TRANSACTIONS OF THE

AMERICAN MATHEMATICAL SOCIETY

Volume 363, Number 12, December 2011, Pages 6421-6437

S 0002-9947(2011)05460-7

Article electronically published on July 22, 2011

\title{
THE DIRICHLET TO NEUMANN OPERATOR FOR ELLIPTIC COMPLEXES
}

\author{
N. TARKHANOV
}

\begin{abstract}
We define the Dirichlet to Neumann operator for an elliptic complex of first order differential operators on a compact Riemannian manifold with boundary. Under reasonable conditions the Betti numbers of the complex prove to be completely determined by the Dirichlet to Neumann operator on the boundary.
\end{abstract}

\section{Contents}

Introduction

1. Bundle decomposition

2. Green formula

3. Hodge theory

4. Poisson formula

5. The "Neumann problem" of Spencer

6. Dirichlet to Neumann operator 6433

7. Betti numbers $\quad 6435$

Acknowledgements $\quad 6436$

References

\section{INTRODUCTION}

Let $\mathcal{X}$ be a connected compact $C^{\infty}$-manifold with boundary equipped with a Riemannian metric. In the sequel $n$ stands for the dimension of $\mathcal{X}$.

Denote by $\Delta$ the Laplace-Beltrami operator on $\mathcal{X}$. The classical Dirichlet to Neumann map $C^{\infty}(\partial \mathcal{X}) \rightarrow C^{\infty}(\partial \mathcal{X})$ is defined by $u_{0} \mapsto \sqrt{-1} n(d u)$, where $u$ is the solution to the Dirichlet problem

$$
\left\{\begin{array}{rlll}
\Delta u & = & 0 & \text { in } \mathcal{X} \\
u & =u_{0} & \text { on } & \partial \mathcal{X}
\end{array}\right.
$$

and $n(d u)$ stands for the normal component of $d u$ on $\partial \mathcal{X}$ which is a constant multiple of the derivative of $u$ along the unit outward normal vector to the boundary. The factor $\sqrt{-1}$ is explained by purely technical reasons. For a deeper discussion

Received by the editors November 23, 2009.

2010 Mathematics Subject Classification. Primary 58J10; Secondary 35R30.

Key words and phrases. Elliptic complexes, Dirichlet to Neumann operator, inverse problems.

(C) 2011 American Mathematical Society 
of the classical Dirichlet to Neumann operator, we refer the reader to Section $12 \mathrm{C}$ of Tay96.

In the inverse problem of reconstructing a manifold from boundary measurements, the following question is of great theoretical and applied interest: To what extent are the topology and geometry of $\mathcal{X}$ determined by the Dirichlet to Neumann map? If $\mathcal{X}$ is of dimension 2 , it proves to be determined by the Dirichlet to Neumann operator up to a conformal equivalence, see [LU01 and Bel03. For $n \geq 3$, there is the conjecture that the Dirichlet to Neumann operator determines $\mathcal{X}$ up to an isometry. In [LTU03] this conjecture is proved for real analytic manifolds $\mathcal{X}$. In the general case it had been known that for $n \geq 3$ the boundary $C^{\infty}$-jet of the Riemannian metric of $\mathcal{X}$ is uniquely determined by the Dirichlet to Neumann map, see [LU89].

In Bel03], an explicit formula is obtained which expresses the Euler characteristic of $\mathcal{X}$ through the Dirichlet to Neumann map in the case of a two-dimensional $\mathcal{X}$ with connected boundary. The Euler characteristic in turn determines the topology of $\mathcal{X}$ in the latter case. The article BSh08 generalises this result to higher dimensions. More precisely, the authors define a Dirichlet to Neumann operator on the space of differential forms of all degrees and express the Betti numbers of $\mathcal{X}$ in terms of this operator.

The Dirichlet to Neumann operator of BSh08 maps differential forms of degree $i$ on $\partial \mathcal{X}$ to those of degree $n-i-1$, i.e., it does not preserve the natural graduation of the space of differential forms. Moreover, as substitution for the Dirichlet problem, the boundary value problem for harmonic forms $u$ on $\mathcal{X}$ is chosen, with prescribed data $t(u)=u_{0}$ and $t\left(d^{*} u\right)=0$ on $\partial \mathcal{X}$, where $t\left(d^{*} u\right)$ is the tangential component of $d^{*} u$ on the boundary.

If specified within general elliptic complexes on $\mathcal{X}$, this substitution is not well motivated for the boundary value problem fails to be elliptic in general. Hence, the Dirichlet to Neumann operator can be defined in this way only for a narrow class of manifolds $\mathcal{X}$.

Here we present another construction of the Dirichlet to Neumann operator for differential forms. To shorten notation, we use the same letter $\Delta$ for the LaplaceBeltrami operator on differential forms in $\mathcal{X}$. By the Dirichlet to Neumann operator is meant the map $\Omega^{i}(\partial \mathcal{X}) \rightarrow \Omega^{i}(\partial \mathcal{X})$ defined by $u_{0} \mapsto \sqrt{-1} n(d u)$, where $u$ is the solution to the problem

$$
\left\{\begin{array}{rlll}
\Delta u & = & \text { in } & \mathcal{X}, \\
t(u) & =u_{0} & \text { on } & \partial \mathcal{X}, \\
n(u) & =0 & \text { on } & \partial \mathcal{X}
\end{array}\right.
$$

on $\mathcal{X}$. Thus, our Dirichlet to Neumann operator preserves the spaces of $i$-forms on $\partial \mathcal{X}$, just as in Bel05. In this way we indeed obtain a straightforward generalisation of $(0.1)$.

This boundary value problem is elliptic and also behaves well in the context of arbitrary elliptic complexes on $\mathcal{X}$. We show that the Dirichlet to Neumann operator defined in this way determines the Betti numbers of $\mathcal{X}$. This is a very particular case of our formula obtained for arbitrary elliptic complexes of first order differential operators on $\mathcal{X}$.

Our contribution is of mainly geometric flavor. There has been recent activity on Dirichlet to Neumann maps, spectral and inverse problems from a more analytic point of view, see [BC10], Hyv09, [KSU07], [NPT07], [NTT09], etc. 


\section{Bundle DeComposition}

Consider an elliptic complex of first order differential operators on $\mathcal{X}$ acting in sections of vector bundles over $\mathcal{X}$. More precisely,

$$
0 \longrightarrow C^{\infty}\left(\mathcal{X}, F^{0}\right) \stackrel{A^{0}}{\longrightarrow} C^{\infty}\left(\mathcal{X}, F^{1}\right) \stackrel{A^{1}}{\longrightarrow} \ldots \stackrel{A^{N-1}}{\longrightarrow} C^{\infty}\left(\mathcal{X}, F^{N}\right) \longrightarrow 0,
$$

where $A^{i} \in \operatorname{Diff}^{1}\left(F^{i}, F^{i+1}\right)$ satisfy $A^{i+1} A^{i}=0$ for all $i$ and the corresponding complex of principal homogeneous symbols is assumed to be exact away from the zero section of $T^{*} \mathcal{X}$. As usual, we write $A^{i} u$ simply as $A u$ for $u \in C^{\infty}\left(\mathcal{X}, F^{i}\right)$ when no confusion can arise.

By $F^{i}$ is meant a smooth vector bundle of rank $k_{i}$ which is nonzero only for $i=0,1, \ldots, N$. We give $F^{i}$ a Hermitian metric, i.e. scalar products $x \mapsto(v, w)_{x}$ in the fibres $F_{x}^{i}$ which smoothly depend on the point $x \in \mathcal{X}$. This defines a conjugate linear isomorphism $*$ of $F^{i}$ to the algebraic dual bundle $F^{i *}$ by $\langle * w, v\rangle_{x}=(v, w)_{x}$ for $v, w \in F_{x}^{i}$.

Fix a smooth positive volume form $d x$ on $\mathcal{X}$. This yields a scalar product on $C^{\infty}\left(\mathcal{X}, F^{i}\right)$ by

$$
(u, v)=\int_{\mathcal{X}}(u(x), v(x))_{x} d x
$$

for $u, v \in C^{\infty}\left(\mathcal{X}, F^{i}\right)$. The completion of this space with respect to the corresponding norm is denoted by $L^{2}\left(\mathcal{X}, F^{i}\right)$. Moreover, we introduce the formal adjoint $A^{i *} \in \operatorname{Diff}^{1}\left(F^{i+1}, F^{i}\right)$ for each operator $A^{i}$ by requiring $(A u, g)=\left(u, A^{*} g\right)$ for all $u \in C^{\infty}\left(\mathcal{X}, F^{i}\right)$ and $g \in C^{\infty}\left(\mathcal{X}, F^{i+1}\right)$ whose supports do not meet each other on the boundary of $\mathcal{X}$.

The operators $\Delta^{i}=A^{i *} A^{i}+A^{i-1} A^{i-1 *}$ are called the Laplacians of (1.1). The ellipticity of this complex is equivalent to the fact that each Laplacian $\Delta^{i} \in \operatorname{Diff}^{2}\left(F^{i}\right)$ is an elliptic operator.

We can assume without loss of generality that $\mathcal{X}$ is embedded into a larger smooth manifold $\mathcal{X}^{\prime}$ without boundary. Choose a smooth function $\varrho$ in a neighbourhood $U$ of $\partial \mathcal{X}$ in $\mathcal{X}^{\prime}$ which is negative in $U \cap(\mathcal{X} \backslash \partial \mathcal{X})$, positive in $U \cap\left(\mathcal{X}^{\prime} \backslash \mathcal{X}\right)$ and whose differential does not vanish on $\partial \mathcal{X}$. By shrinking $U$ if necessary, we may actually assume that $|d \varrho(x)|=1$ holds for all $x \in \partial \mathcal{X}$, for if not, we replace $\varrho$ by $\varrho /|d \varrho|$.

Lemma 1.1. For $x \in \partial \mathcal{X}$, the cotangent vector $d \varrho(x) \in T_{x}^{*} \mathcal{X}$ is independent of the particular choice of $\varrho$.

Proof. Let $\varrho_{1}$ and $\varrho_{2}$ be two functions with the properties described above. For each $x \in \partial \mathcal{X}$ there is a neighbourhood $U_{x}$ of this point in $\mathcal{X}^{\prime}$, such that $\varrho_{2}=f \varrho_{1}$ in $U_{x}$ with some smooth function $f$ in $U_{x}$. It is clear that $f$ is positive in $U_{x} \backslash \partial \mathcal{X}$. Furthermore, we get $d \varrho_{2}=f d \varrho_{1}$ on $U_{x} \cap \partial \mathcal{X}$, whence $f \equiv 1$ on $U_{x} \cap \partial \mathcal{X}$, as desired.

Write $\sigma^{i}(x)$ for the principal homogeneous symbol of $A^{i}$ evaluated at the point $(x, d \varrho(x))$ of $T^{*} \mathcal{X}$. This is a smooth section of the bundle $\operatorname{Hom}\left(F^{i}, F^{i+1}\right)$ whose restriction to $\partial \mathcal{X}$ does not depend on the choice of $\varrho$, the latter being due to Lemma 1.1] The principal homogeneous symbol of $\Delta^{i}$ evaluated at $(x, d \varrho(x))$ is $\sigma^{i}(x)^{*} \sigma^{i}(x)+\sigma^{i-1}(x) \sigma^{i-1}(x)^{*}$, which we denote by $\ell^{i}(x)$ for short. Since $\Delta^{i}$ is elliptic, the map $\ell^{i}(x) \in \operatorname{Hom}\left(F_{x}^{i}\right)$ is invertible in some neighbourhood of the hypersurface $\partial \mathcal{X}$ in $\mathcal{X}^{\prime}$. 
Lemma 1.2. For every $i$, the restriction of the bundle $F^{i}$ to $\partial \mathcal{X}$ splits into the direct sum

$$
F^{i} \uparrow_{\partial \mathcal{X}}=F_{t}^{i} \oplus \sigma^{i-1} F_{t}^{i-1}
$$

where $F_{t}^{i}=\sigma^{i *} \sigma^{i}\left(\ell^{i}\right)^{-1} F^{i} \uparrow_{\partial \mathcal{X}}$ is a smooth subbundle of $F^{i}\lceil\partial \mathcal{X}$.

Proof. For each $x \in \mathcal{X}$ close to the boundary, any $v \in F_{x}^{i}$ can be written in the form

$$
v=t(v)+\sigma^{i-1}(x) n(v)
$$

where

$$
\begin{aligned}
t(v) & =\sigma^{i}(x)^{*} \sigma^{i}(x)\left(\ell^{i}(x)\right)^{-1} v, \\
n(v) & =\sigma^{i-1}(x)^{*}\left(\ell^{i}(x)\right)^{-1} v
\end{aligned}
$$

prove to satisfy $t \circ t=t, t \circ n=n, n \circ t=0$ and $n \circ n=0$. This establishes the lemma.

Note that if $F^{i}=\Lambda^{i} T^{*} \mathcal{X}$ is the bundle of exterior forms of degree $i$ over $\mathcal{X}$, then $F_{t}^{i}=\iota^{*} F^{i}$ is the pullback of $F^{i}$ under the embedding $\partial \mathcal{X} \hookrightarrow \mathcal{X}$. It follows that $F_{t}^{i}=\Lambda^{i} T^{*}(\partial \mathcal{X})$.

\section{Green formula}

To describe natural boundary value problems for solutions of $\Delta^{i} u=f$ in $\mathcal{X}$, one uses a Green formula related to the Laplacian $\Delta^{i}$. Such formulas are well understood in general, see for instance Lemma 3.2.10 in Tar95]. In this section we just compute explicitly the terms included in this formula, to get it in the form we need.

Lemma 2.1 (Green formula). For each $u, v \in H^{2}\left(\mathcal{X}, F^{i}\right)$, the equality

$$
\begin{aligned}
& \int_{\partial \mathcal{X}}\left((t(u), \imath \ell n(A v))_{x}-\left(\imath \ell n(u), t\left(A^{*} v\right)\right)_{x}+\left(t\left(A^{*} u\right), \imath \ln (v)\right)_{x}-(\imath \ln (A u), t(v))_{x}\right) d s \\
& \quad=\int_{\mathcal{X}}\left((\Delta u, v)_{x}-(u, \Delta v)_{x}\right) d x
\end{aligned}
$$

is true, where $d s$ is the surface measure on $\partial \mathcal{X}$ induced by the volume form $d x$ in $\mathcal{X}$, and $\imath=\sqrt{-1}$.

Proof. Let $G_{A}(* g, u)$ be the Green operator for a differential operator $A=A^{i}$, see $\S 2.4 .2$ of [Tar95]. An easy computation shows that the pullbacks of differential forms $G_{A}(* g, u)$ and $G_{A^{*}}(* u, g)$ under the inclusion mapping $\partial \mathcal{X} \hookrightarrow \mathcal{X}$ just amount to

$$
\begin{aligned}
\iota^{*} G_{A}(* g, u) & =(t(u), \imath \ell n(g))_{x} d s, \\
\iota^{*} G_{A^{*}}(* u, g) & =-(\imath \ell n(g), t(u))_{x} d s
\end{aligned}
$$

on $\partial \mathcal{X}$ for all smooth sections $g$ and $u$ of $F^{i+1}$ and $F^{i}$, respectively, cf. $\S 3.2 .2$ ibid. Applying Corollary 2.5.14 of [Tar95] establishes the formula.

It follows that the quadruple $t(u), n(u), t\left(A^{*} u\right)$ and $n(A u)$ gives a representation of the Cauchy data of $u$ on $\partial \mathcal{X}$ relative to the Laplacian $\Delta$. The tangential part of the Cauchy data, $\left(t(u), t\left(A^{*} u\right)\right)$, is usually referred to as the Dirichlet data, and the normal part of the Cauchy data, $(n(u), n(A u))$, is referred to as the Neumann data. This designation is due to the whimsical development of mathematics rather than to well-motivated choice, for $\left(t(u), t\left(A^{*} u\right)\right)$ reduces to $t\left(A^{*} u\right)$ at the last step of the 
complex, which is the classical Neumann data, and $(n(u), n(A u))$ reduces to $n(u)$ at the first step of the complex, which is the classical Dirichlet data. Moreover, the Dirichlet data relative to the complex (1.1) just amount to the Neumann data for the formally adjoint complex.

\section{Hodge THEORY}

Given a vector space $V$ with norm $\|\cdot\|$, we write $C(V,\|\cdot\|)$ for the completion of $V$ under the norm $\|\cdot\|$.

In this section we describe the Hodge theory of the Dirichlet problem for the Laplacian $\Delta^{i}$ in the class of generalised sections of $F^{i}$ on $\mathcal{X}$. In order to define what is meant by the "solution" of the boundary value problem, we employ negative norms. This idea is certainly not new and goes back at least as far as Sch60 and Roi96.

Given a section $f$ of $F^{i}$, we consider the boundary value problem

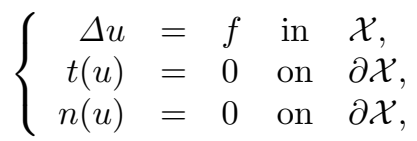

which is an obvious generalisation of the classical Dirichlet problem. This latter corresponds to the de Rham complex and $i=0$. Obviously, the conditions $t(u)=0$ and $n(u)=0$ on $\partial \mathcal{X}$ are equivalent to the fact that $u=0$ on $\partial \mathcal{X}$.

Suppose $s>0$. For sections $u \in C^{\infty}\left(\mathcal{X}, F^{i}\right)$ we introduce two types of negative norms:

$$
\begin{aligned}
\|u\|_{-s} & =\sup _{v \in C^{\infty}\left(\mathcal{X}, F^{i}\right)} \frac{|(u, v)|}{\|v\|_{s}}, \\
|u|_{-s} & =\sup _{\substack{v \in C^{\infty}\left(\mathcal{X}, F^{i}\right) \\
t(v)=0 \\
n(v)=0}} \frac{|(u, v)|}{\|v\|_{s}},
\end{aligned}
$$

where $(\cdot, \cdot)$ is the scalar product in $L^{2}\left(\mathcal{X}, F^{i}\right)$. We denote the completions of $C^{\infty}\left(\mathcal{X}, F^{i}\right)$ with respect to these norms by $H^{-s}\left(\mathcal{X}, F^{i}\right)$ and $C\left(C^{\infty}\left(\mathcal{X}, F^{i}\right),|\cdot|_{-s}\right)$, respectively. They are obviously Banach spaces and satisfy

$$
H^{-s}\left(\mathcal{X}, F^{i}\right) \hookrightarrow C\left(C^{\infty}\left(\mathcal{X}, F^{i}\right),|\cdot|_{-s}\right),
$$

for $\|u\|_{-s} \geq|u|_{-s}$.

We can define $(u, v)$ for $u \in H^{-s}\left(\mathcal{X}, F^{i}\right)$ and $v \in C^{\infty}\left(\mathcal{X}, F^{i}\right)$ as follows. By definition, there is a sequence $\left\{u_{\nu}\right\}$ in $C^{\infty}\left(\mathcal{X}, F^{i}\right)$ such that $\left\|u_{\nu}-u\right\|_{-s} \rightarrow 0$ as $\nu \rightarrow \infty$. Then

$$
\begin{aligned}
\left|\left(u_{\nu}-u_{\mu}, v\right)\right| & \leq\left\|u_{\nu}-u_{\mu}\right\|_{-s}\|v\|_{s} \\
& \rightarrow 0
\end{aligned}
$$

as $\mu, \nu \rightarrow \infty$. Set $(u, v)=\lim \left(u_{\nu}, v\right)$. Clearly, this limit does not depend on the particular sequence $\left\{u_{\nu}\right\}$, for if $\left\|u_{\nu}\right\|_{-s} \rightarrow 0$, then $\left|\left(u_{\nu}, v\right)\right| \leq\left\|u_{\nu}\right\|_{-s}\|v\|_{s}$ tends to zero, too. From the definition it follows that for all sections $u \in H^{-s}\left(\mathcal{X}, F^{i}\right)$ and $v \in C^{\infty}\left(\mathcal{X}, F^{i}\right)$ we get

$$
|(u, v)| \leq\|u\|_{-s}\|v\|_{s}
$$


In a similar way we can define the pairing $(u, v)$ for $u \in C\left(C^{\infty}\left(\mathcal{X}, F^{i}\right),|\cdot|_{-s}\right)$ and $v \in C^{\infty}\left(\mathcal{X}, F^{i}\right)$ with $t(v)=0$ and $n(v)=0$. Corresponding to (3.2) we obviously have $|(u, v)| \leq|u|_{-s}\|v\|_{s}$.

Suppose that $f$ is in $C\left(C^{\infty}\left(\mathcal{X}, F^{i}\right),|\cdot|_{-s-2}\right)$, where $s \geq 0$. We shall say that $u \in H^{-s}\left(\mathcal{X}, F^{i}\right)$ is a strong solution of (3.1) if there is a sequence of sections $u_{\nu} \in C^{\infty}\left(\mathcal{X}, F^{i}\right)$ with $t\left(u_{\nu}\right)=0$ and $n\left(u_{\nu}\right)=0$ such that

$$
\begin{aligned}
\left\|u_{\nu}-u\right\|_{-s} & \rightarrow 0 \\
\left|\Delta u_{\nu}-f\right|_{-s-2} & \rightarrow 0
\end{aligned}
$$

as $\nu \rightarrow \infty$.

Denote by $\mathcal{H}^{i}(\mathcal{X})$ the set of all $u \in C^{\infty}\left(\mathcal{X}, F^{i}\right)$ that satisfy $\Delta u=0$ in the interior of $X$ and $t(u)=0$ and $n(u)=0$ on $\partial X$. Since (3.1) is an elliptic boundary value problem, $\mathcal{H}^{i}(\mathcal{X})$ is finite dimensional. Moreover, for any $u \in \mathcal{H}^{i}(\mathcal{X})$ we actually obtain

$$
\begin{aligned}
0 & =(\Delta u, u) \\
& =(A u, A u)+\left(A^{*} u, A^{*} u\right),
\end{aligned}
$$

whence $A u=0$ and $A^{*} u=0$ in $\mathcal{X}$. Therefore, the space $\mathcal{H}(\mathcal{X})$ consists of all solutions to the overdetermined elliptic system $A u=0$ and $A^{*} u=0$ in the interior of $\mathcal{X}$ which are $C^{\infty}$ up to the boundary of $X$ and which vanish up to the infinite order on $\partial \mathcal{X}$.

Lemma 3.1. Let $s \geq 0$. If $f \in C\left(C^{\infty}\left(\mathcal{X}, F^{i}\right),|\cdot|_{-s-2}\right)$ and $f \perp \mathcal{H}^{i}(\mathcal{X})$, then there is a strong solution $u \in H^{-s}\left(\mathcal{X}, F^{i}\right)$ of (3.1) satisfying $u \perp \mathcal{H}^{i}(\mathcal{X})$ and

$$
\|u\|_{-s} \leq c|f|_{-s-2},
$$

where the constant $c$ does not depend on $f$ and $u$.

Proof. Cf. [Sch60].

The definition (3.3) of a strong solution to Dirichlet problem (3.1) obviously corresponds to an appropriate closure $L: \mathcal{D}_{L^{i}} \rightarrow C\left(C^{\infty}\left(\mathcal{X}, F^{i}\right),|\cdot|_{-s-2}\right)$ of the Laplacian $\Delta=A^{*} A+A A^{*}$. More precisely, we denote by $\mathcal{D}_{L^{i}}$ the set of all sections $u \in H^{-s}\left(\mathcal{X}, F^{i}\right)$, for which there exists a sequence $\left\{u_{\nu}\right\}$ with the following properties:

1) $u_{\nu} \in C^{\infty}\left(\mathcal{X}, F^{i}\right)$ satisfies $t\left(u_{\nu}\right)=0$ and $n\left(u_{\nu}\right)=0$;

2) $\left\{u_{\nu}\right\}$ converges to $u$ in $H^{-s}\left(\mathcal{X}, F^{i}\right)$;

3) $\left\{\Delta u_{\nu}\right\}$ is a Cauchy sequence in $C\left(C^{\infty}\left(\mathcal{X}, F^{i}\right),|\cdot|_{-s-2}\right)$.

The closed densely defined operator $L: \mathcal{D}_{L^{i}} \rightarrow C\left(C^{\infty}\left(\mathcal{X}, F^{i}\right),|\cdot|_{-s-2}\right)$ given by $L u=\lim \Delta u_{\nu}$, where $\left\{u_{\nu}\right\}$ is any sequence with properties 1)-3), is called the strong extension of $\Delta$ under the boundary conditions $t(u)=0$ and $n(u)=0$. It is clear that $u \in H^{-s}\left(\mathcal{X}, F^{i}\right)$ is a strong solution to problem (3.1) if and only if $L u=f$.

It is worth pointing out that the case $\partial \mathcal{X}=\emptyset$ is formally permitted in Theorem 3.2 below. In this way we generalise the Hodge theory for elliptic complexes on smooth compact closed manifolds to the case of smooth compact manifolds with boundary.

Theorem 3.2. Suppose $s \geq 0$. There are bounded linear operators

$$
\begin{aligned}
& H: \quad C\left(C^{\infty}\left(\mathcal{X}, F^{i}\right),|\cdot|_{-s-2}\right) \rightarrow \mathcal{H}^{i}(\mathcal{X}), \\
& G: \quad C\left(C^{\infty}\left(\mathcal{X}, F^{i}\right),|\cdot|_{-s-2}\right) \rightarrow \mathcal{D}_{L^{i}}
\end{aligned}
$$


such that

1) $H^{i}$ has the kernel $K_{H^{i}}\left(x, x^{\prime}\right)=\sum_{\nu} h_{i, \nu}(x) \otimes * h_{i, \nu}\left(x^{\prime}\right)$, where $\left\{h_{i, \nu}\right\}$ is an orthonormal basis of $\mathcal{H}^{i}(\mathcal{X})$;

2) $A H=H A=0$ and $G H=H G=0$;

3)

$$
\begin{aligned}
& G L u=u-H u \text { for all } u \in \mathcal{D}_{L^{i}}, \\
& L G f=f-H f \text { for all } f \in C\left(C^{\infty}\left(\mathcal{X}, F^{i}\right),|\cdot|_{-s-2}\right) .
\end{aligned}
$$

Proof. This follows by the same method as in Theorem 3.3 of [SST03, with Lemma 3.2 there replaced by Lemma 3.1 .

The operators $H$ and $G$ are actually independent of $s$ since they are unique extensions by continuity of these operators on the dense subspace $C^{\infty}\left(\mathcal{X}, F^{i}\right)$ of $C\left(C^{\infty}\left(\mathcal{X}, F^{i}\right),|\cdot|{ }_{-s-2}\right)$.

When restricted to $L^{2}\left(\mathcal{X}, F^{i}\right)$, the operator $G$ is selfadjoint. Indeed, given any $f, v \in L^{2}\left(\mathcal{X}, F^{i}\right)$, we may invoke the elliptic regularity of the Dirichlet problem (3.1) to conclude that both $G f$ and $G v$ belong to $H^{2}\left(\mathcal{X}, F^{i}\right)$ and satisfy the boundary conditions $t(\cdot)=0$ and $n(\cdot)=0$. It follows that $L G f=\Delta G f$ and $L G v=\Delta G v$, whence

$$
\begin{aligned}
(G f, v) & =(G f, H v+L G v) \\
& =\left(G f,\left(A^{*} A+A A^{*}\right) G v\right) \\
& =\left(\left(A^{*} A+A A^{*}\right) G f, G v\right) \\
& =(f, G v),
\end{aligned}
$$

which is due to Theorem 3.2. Hence the Schwartz kernel of $G$ is Hermitian, i.e., $K_{G}(x, y)^{*}=K_{G}(y, x)$ for all $(x, y)$ away from the diagonal of $\mathcal{X} \times \mathcal{X}$.

Corollary 3.3. If, in addition, $f \in H^{-s-2}\left(\mathcal{X}, F^{i}\right)$, then there is a sequence of sections $u_{\nu} \in C^{\infty}\left(\mathcal{X}, F^{i}\right)$ with $t\left(u_{\nu}\right)=0$ and $n\left(u_{\nu}\right)=0$, such that

$$
\begin{aligned}
\left\|u_{\nu}-u\right\|_{-s} & \rightarrow 0 \\
\left\|\Delta u_{\nu}-f\right\|_{-s-2} & \rightarrow 0
\end{aligned}
$$

as $\nu \rightarrow \infty$.

From Lemma 2.1 we deduce that when $u$ is smooth enough, it fulfills $t(u)=0$ and $n(u)=0$ if and only if $(\Delta u, v)=(u, \Delta v)$ for all $v$ satisfying $t(v)=0$ and $n(v)=0$. This gives rise to the concept of a weak extension of $\Delta$ under the boundary conditions $t(u)=0$ and $n(u)=0$. Given an $f \in C\left(C^{\infty}\left(\mathcal{X}, F^{i}\right),|\cdot|{ }_{-s-2}\right)$, a section $u$ is said to be a weak solution of (3.1) if it is in $H^{-s^{\prime}}\left(\mathcal{X}, F^{i}\right)$ for some $s^{\prime} \geq 0$ and

$$
(u, \Delta v)=(f, v)
$$

for all $v \in C^{\infty}\left(\mathcal{X}, F^{i}\right)$ satisfying $t(v)=0$ and $n(v)=0$.

Lemma 3.4. Suppose $f \in C\left(C^{\infty}\left(\mathcal{X}, F^{i}\right),|\cdot|_{-s-2}\right)$, where $s \geq 0$. If $u \in H^{-s^{\prime}}\left(\mathcal{X}, F^{i}\right)$ is a weak solution of (3.1), then $u$ actually belongs to $H^{-s}\left(\mathcal{X}, F^{i}\right)$ and it is a strong solution of (3.1). Moreover, there is a constant $c$ not depending on $f$ or $u$, such that

$$
\|u\|_{-s} \leq c\left(|f|_{-s-2}+\|u\|_{-s^{\prime}}\right) .
$$

Proof. Cf. Sch60]. 


\section{Poisson Formula}

To study the Dirichlet problem with nonzero boundary data $t(u)=u_{0,1}$ and $n(u)=u_{0,2}$ we need a result of Roi96. Namely, motivated by the Green formula for the Laplacian $\Delta^{i}$ we denote by $H^{-s, B}\left(\mathcal{X}, F^{i}\right)$ the completion of $C^{\infty}\left(\mathcal{X}, F^{i}\right)$ with respect to the norm

$$
\begin{aligned}
\|u\|_{-s, B}:= & \|u\|_{-s}+\|t(u)\|_{H^{-s-1 / 2}\left(\partial \mathcal{X}, F_{t}^{i}\right)}+\|n(u)\|_{H^{-s-1 / 2}\left(\partial \mathcal{X}, F_{t}^{i-1}\right)} \\
& +\left\|t\left(A^{*} u\right)\right\|_{H^{-s-3 / 2}\left(\partial \mathcal{X}, F_{t}^{i-1}\right)}+\|n(A u)\|_{H^{-s-3 / 2}\left(\partial \mathcal{X}, F_{t}^{i}\right)},
\end{aligned}
$$

cf. Lemma 2.1. The advantage of using these spaces lies in the fact that for each $u \in H^{-s, B}\left(\mathcal{X}, F^{i}\right)$ there is a sequence $\left\{u_{\nu}\right\}$ in $C^{\infty}\left(\mathcal{X}, F^{i}\right)$, such that $u_{\nu} \rightarrow u$ in $H^{-s}\left(\mathcal{X}, F^{i}\right)$ and $\left\{t\left(u_{\nu}\right)\right\},\left\{n\left(u_{\nu}\right)\right\},\left\{t\left(A^{*} u_{\nu}\right)\right\},\left\{n\left(A u_{\nu}\right)\right\}$ are Cauchy sequences in $H^{-s-1 / 2}\left(\partial \mathcal{X}, F_{t}^{i}\right), H^{-s-1 / 2}\left(\partial \mathcal{X}, F_{t}^{i-1}\right), H^{-s-3 / 2}\left(\partial \mathcal{X}, F_{t}^{i-1}\right)$ and $H^{-s-3 / 2}\left(\partial \mathcal{X}, F_{t}^{i}\right)$, respectively. Moreover, $\left\{\Delta u_{\nu}\right\}$ is a Cauchy sequence in $H^{-s-2}\left(\mathcal{X}, F^{i}\right)$, which follows by manipulations of the Green formula. Hence to any element $u \in$ $H^{-s, B}\left(\mathcal{X}, F^{i}\right)$ we can assign both $t(u), n(u), t\left(A^{*} u\right), n(A u)$ and $\Delta u$ defined in the above strong sense.

Lemma 4.1. For each quadruple

$$
\begin{array}{lll}
u_{0,1} \in H^{-s-1 / 2}\left(\partial \mathcal{X}, F_{t}^{i}\right), & u_{0,2} \in H^{-s-1 / 2}\left(\partial \mathcal{X}, F_{t}^{i-1}\right), \\
u_{1,1} \in H^{-s-3 / 2}\left(\partial \mathcal{X}, F_{t}^{i-1}\right), & u_{1,2} \in H^{-s-3 / 2}\left(\partial \mathcal{X}, F_{t}^{i}\right),
\end{array}
$$

there is $u \in H^{-s, B}\left(\mathcal{X}, F^{i}\right)$, such that $t(u)=u_{0,1}, n(u)=u_{0,2}, t\left(A^{*} u\right)=u_{1,1}$ and $n(A u)=u_{1,2}$ on $\partial \mathcal{X}$. Moreover, the map $\left(u_{0,1}, u_{0,2}, u_{1,1}, u_{1,2}\right) \mapsto u$ is continuous in the relevant norms.

Proof. For smooth Cauchy data $u_{0,1}, u_{0,2}, u_{1,1}$, and $u_{1,2}$ a stronger result is contained in Lemma 3.2.9 of [Tar95]. For the general case, see Lemma 6.1.2 in Roi96.

Given any $f \in C\left(C^{\infty}\left(\mathcal{X}, F^{i}\right),|\cdot|_{-s-2}\right)$ and

$$
\begin{aligned}
& u_{0,1} \in H^{-s-1 / 2}\left(\partial \mathcal{X}, F_{t}^{i}\right), \\
& u_{0,2} \in H^{-s-1 / 2}\left(\partial \mathcal{X}, F_{t}^{i-1}\right),
\end{aligned}
$$

we now consider the inhomogeneous Dirichlet problem

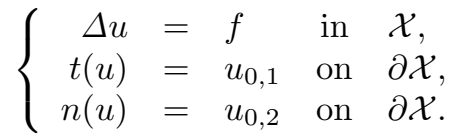

A section $u$ is said to be a weak solution of (4.2) if it is in $H^{-s^{\prime}}\left(\mathcal{X}, F^{i}\right)$ for some $s^{\prime} \geq 0$ and

$$
(u, \Delta v)=(f, v)-\int_{\partial \mathcal{X}}\left(\left(u_{0,1}, \iota \ell n(A v)\right)_{x}-\left(\iota \ell u_{0,2}, t\left(A^{*} v\right)\right)_{x}\right) d s
$$

for all $v \in C^{\infty}\left(\mathcal{X}, F^{i}\right)$ satisfying $t(v)=0$ and $n(v)=0$.

Theorem 4.2. Suppose $s \geq 0$. If $f \perp \mathcal{H}^{i}(\mathcal{X})$, then there is a weak solution $u \in H^{-s^{\prime}}\left(\mathcal{X}, F^{i}\right)$ to (4.2) with $u \perp \mathcal{H}^{i}(\mathcal{X})$. Moreover, $u \in H^{-s}\left(\mathcal{X}, F^{i}\right)$ satisfies (4.2) in a strong sense, and there is a constant $c$ independent of $f, u_{0,1}, u_{0,2}$ and $u$, such that

$$
\|u\|_{-s} \leq c\left(|f|_{-s-2 m}+\left\|u_{0,1}\right\|_{H^{-s-1 / 2}\left(\partial \mathcal{X}, F_{t}^{i}\right)}+\left\|u_{0,2}\right\|_{H^{-s-1 / 2}\left(\partial \mathcal{X}, F_{t}^{i-1}\right)}\right) .
$$


Proof. Using Lemma 4.1 we reduce (4.3) to (3.6) with a suitable right side $f$. To this end we choose $U \in H^{-s, B}\left(\mathcal{X}, F^{i}\right)$ such that $t(U)=u_{0,1}, n(U)=u_{0,2}$ and $t\left(A^{*} U\right)=u_{1,1}, n(A U)=u_{1,2}$, where $u_{1,1}$ and $u_{1,2}$ are arbitrary. By the above, there exists a sequence $\left\{u_{\nu}\right\}$ in $C^{\infty}\left(\mathcal{X}, F^{i}\right)$ converging to $U$ in $H^{-s}\left(\mathcal{X}, F^{i}\right)$, such that

$$
\begin{aligned}
t\left(u_{\nu}\right) & \rightarrow u_{0,1} \text { in } H^{-s-1 / 2}\left(\partial \mathcal{X}, F_{t}^{i}\right), & n\left(u_{\nu}\right) & \rightarrow u_{0,2} \text { in } H^{-s-1 / 2}\left(\partial \mathcal{X}, F_{t}^{i-1}\right), \\
t\left(A^{*} u_{\nu}\right) & \rightarrow u_{1,1} \text { in } H^{-s-3 / 2}\left(\partial \mathcal{X}, F_{t}^{i-1}\right), & n\left(A u_{\nu}\right) & \rightarrow u_{1,2} \text { in } H^{-s-3 / 2}\left(\partial \mathcal{X}, F_{t}^{i}\right)
\end{aligned}
$$

and $\Delta u_{\nu} \rightarrow f^{\prime}$ in $H^{-s-2}\left(\mathcal{X}, F^{i}\right)$.

By the Green formula, we get

$$
\left(u_{\nu}, \Delta v\right)=\left(\Delta u_{\nu}, v\right)-\int_{\partial \mathcal{X}}\left(\left(t\left(u_{\nu}\right), \iota \ell n(A v)\right)_{x}-\left(\iota \ell n\left(u_{\nu}\right), t\left(A^{*} v\right)\right)_{x}\right) d s
$$

for all $v \in C^{\infty}\left(\mathcal{X}, F^{i}\right)$ satisfying $t(v)=0$ and $n(v)=0$. Letting $\nu \rightarrow \infty$ in this equality yields

$$
\left.(U, \Delta v)=\left(f^{\prime}, v\right)-\int_{\partial \mathcal{X}}\left(u_{0,1}, \iota \ell n(A v)\right)_{x}-\left(\iota \ell u_{0,2}, t\left(A^{*} v\right)\right)_{x}\right) d s .
$$

Subtracting (4.5) from (4.3) we obtain

$$
(u-U, \Delta v)=\left(f-f^{\prime}, v\right)
$$

for all $v \in C^{\infty}\left(\mathcal{X}, F^{i}\right)$ satisfying $t(v)=0$ and $n(v)=0$, i.e., $u-U$ is a weak solution of the Dirichlet problem (3.1) with $f$ replaced by $f-f^{\prime}$. Moreover, it follows from (4.5) that

$$
\left(f^{\prime}, v\right)=0
$$

for all $v \in \mathcal{H}^{i}(\mathcal{X})$. Combining Lemmas 3.4 and 3.1 thus results in the desired assertion.

We now derive a Poisson formula for solutions of the inhomogeneous Dirichlet problem.

For $A=A^{i}$, we denote by $[\partial \mathcal{X}]^{A}$ the kernel on $\mathcal{X} \times \mathcal{X}$ defined by

$$
\left\langle[\partial \mathcal{X}]^{A}, * g \otimes u\right\rangle=\int_{\partial \mathcal{X}} G_{A}(* g, u)
$$

for all $g \in C^{\infty}\left(\mathcal{X}, F^{i+1}\right)$ and $u \in C^{\infty}\left(\mathcal{X}, F^{i}\right)$. It is obviously supported on the diagonal of $\partial \mathcal{X} \times \partial \mathcal{X}$.

Given any $u \in C^{\infty}\left(\mathcal{X}, F^{i}\right)$, we set

$$
\begin{aligned}
(M u)(x) & =-G A^{*}\left([\partial X]^{A} u\right)-G A\left([\partial X]^{A^{*}} u\right) \\
& =-\int_{\partial \mathcal{X}}\left(\left(t(u), \iota \ell n\left(*^{-1} K_{G A^{*}}(x, \cdot)\right)\right)_{x^{\prime}}-\left(\iota \ell n(u), t\left(*^{-1} K_{G A}(x, \cdot)\right)\right)_{x^{\prime}}\right) d s
\end{aligned}
$$

for $x$ in the interior of $X$, where $K_{G A^{*}}$ is the Schwartz kernel of $G A^{*}$, and similarly for $K_{G A}$. The integral on the right-hand side is well defined, for $K_{G A^{*}}$ and $K_{G A}$ are smooth sections of $F^{i} \otimes F^{i+1 *}$ and $F^{i} \otimes F^{i-1 *}$ outside of the diagonal of $\mathcal{X} \times \mathcal{X}$, respectively.

Corollary 4.3. As defined above, $M$ induces a continuous map $P$ of the product $H^{-s-1 / 2}\left(\partial \mathcal{X}, F_{t}^{i}\right) \times H^{-s-1 / 2}\left(\partial \mathcal{X}, F_{t}^{i-1}\right)$ to $H^{-s}\left(\mathcal{X}, F^{i}\right)$ with the property that 
$P(t(u), n(u))=M u$ for all $u \in C^{\infty}\left(\mathcal{X}, F^{i}\right)$. Moreover, for each weak solution $u$ of (4.2) it follows that

$$
u=H u+G \Delta u+P(t(u), n(u)) .
$$

Proof. Let $u \in H^{-s^{\prime}}\left(\mathcal{X}, F^{i}\right)$ be a weak solution to (4.2). From Theorem 4.2 we readily deduce that $u \in H^{-s}\left(\mathcal{X}, F^{i}\right)$ satisfies (4.2) in a strong sense. More precisely, there exists a sequence $u_{\nu} \in C^{\infty}\left(\mathcal{X}, F^{i}\right)$ which approximates $u$ in $H^{-s}\left(\mathcal{X}, F^{i}\right)$, such that $t\left(u_{\nu}\right) \rightarrow t(u), n\left(u_{\nu}\right) \rightarrow n(u)$ and $\Delta u_{\nu} \rightarrow \Delta u$ in the relevant norms. We now set

$$
\begin{aligned}
P\left(u_{0,1}, u_{0,2}\right) & :=\lim _{\nu \rightarrow \infty}\left(u_{\nu}-H u_{\nu}-G\left(\Delta u_{\nu}\right)\right) \\
& =u-H u-G(\Delta u) ;
\end{aligned}
$$

the limit exists in $H^{-s}\left(\mathcal{X}, F^{i}\right)$ by Theorem 3.2. Moreover, it is independent of the particular choice of $u$ with well-defined $\Delta u$ and $t(u)=u_{0,1}, n(u)=u_{0,2}$, which is again due to Theorem 3.2 .

Obviously, $\left(u_{0,1}, u_{0,2}\right) \mapsto P\left(u_{0,1}, u_{0,2}\right)$ is a continuous mapping of the product $H^{-s-1 / 2}\left(\partial \mathcal{X}, F_{t}^{i}\right) \times H^{-s-1 / 2}\left(\partial \mathcal{X}, F_{t}^{i-1}\right)$ to $H^{-s}\left(\mathcal{X}, F^{i}\right)$, and it remains to prove that $P\left(u_{0,1}, u_{0,2}\right)$ actually agrees to $-G A^{*}\left([\partial \mathcal{X}]^{A} u\right)-G A\left([\partial \mathcal{X}]^{A^{*}} u\right)$ in the interior of $\mathcal{X}$.

If $v \in C^{\infty}\left(\mathcal{X}, F^{i}\right)$ has a compact support in the interior of $\mathcal{X}$, then by Theorem 3.2 we get

$$
\begin{aligned}
& \left(P\left(u_{0,1}, u_{0,2}\right), v\right)=(u, v)-(u, H v)-(\Delta u, G v) \\
& \quad=(u, v-H v-\Delta(G v))-\int_{\partial \mathcal{X}}\left((t(u), \iota \ln (A G v))_{x}-\left(\iota \ln (u), t\left(A^{*} G v\right)\right)_{x}\right) d s \\
& =-\int_{\partial \mathcal{X}}\left((t(u), \iota \ln (A G v))_{x}-\left(\iota \ln (u), t\left(A^{*} G v\right)\right)_{x}\right) d s,
\end{aligned}
$$

for $t(G v)=0$ and $n(G v)=0$. The right-hand side here just amounts to the scalar product $\left(-G A^{*}\left([\partial \mathcal{X}]^{A} u\right)-G A\left([\partial \mathcal{X}]^{A^{*}} u\right), v\right)$, provided that $u$ is smooth enough.

From (4.7) it follows that $P\left(u_{0,1}, u_{0,2}\right)$ is the unique solution to the Dirichlet problem (4.2) with zero data $f \equiv 0$ in $\mathcal{X}$ that is orthogonal to $\mathcal{H}^{i}(\mathcal{X})$. We call $P\left(u_{0,1}, u_{0,2}\right)$ the Poisson integral of boundary data $\left(u_{0,1}, u_{0,2}\right)$. Theorem 4.2 shows that

$$
\left\|P\left(u_{0,1}, u_{0,2}\right)\right\|_{-s} \leq c\left(\left\|u_{0,1}\right\|_{H^{-s-1 / 2}\left(\partial \mathcal{X}, F_{t}^{i}\right)}+\left\|u_{0,2}\right\|_{H^{-s-1 / 2}\left(\partial \mathcal{X}, F_{t}^{i-1}\right)}\right)
$$

holds with $c$ a constant independent of $u_{0,1}$ and $u_{0,2}$.

\section{The "Neumann problem" of Spencer}

As developed in Section 3. the Hodge theory for elliptic complexes on manifolds with boundary falls short of providing any information on the cohomology of the complex (1.1). The main difficulty in carrying out this study is that $A G f$ need not vanish if $A f=0$.

The "Neumann problem" of Spencer is motivated by the problem of solvability of the inhomogeneous equation $A y=f$ in $\mathcal{X}$. Given section $f \in C^{\infty}\left(\mathcal{X}, F^{i}\right)$, under what conditions does there exist a section $y \in C^{\infty}\left(\mathcal{X}, F^{i-1}\right)$ satisfying $A y=f$, and how does $y$ depend on $f$ ? 
Lemma 5.1. In order that $A y=f$ may be solvable, it is necessary that $(f, g)=0$ for all $g \in C^{\infty}\left(\mathcal{X}, F^{i}\right)$ satisfying $A^{*} g=0$ in $\mathcal{X}$ and $n(g)=0$ on $\partial \mathcal{X}$.

Proof. Indeed, if $y \in C^{\infty}\left(\mathcal{X}, F^{i-1}\right)$ and $g \in C^{\infty}\left(\mathcal{X}, F^{i}\right)$, then we have by Stokes' formula,

showing the lemma.

$$
(A y, g)=\left(y, A^{*} g\right)+\int_{\partial \mathcal{X}}(t(y), \imath \ell n(g))_{x} d s
$$

Denote by $\mathcal{H}_{N}^{i}(\mathcal{X})$ the space of all $h \in C^{\infty}\left(\mathcal{X}, F^{i}\right)$ with the property that $A h=0$ and $A^{*} h=0$ in $\mathcal{X}$ and $n(h)=0$ on $\partial \mathcal{X}$. We give $\mathcal{H}_{N}^{i}(\mathcal{X})$ the topology induced from $C^{\infty}\left(\mathcal{X}, F^{i}\right)$. The elements of $\mathcal{H}_{N}^{i}(\mathcal{X})$ are called harmonic sections of $F^{i}{ }_{\lceil\mathcal{X}}$ for the "Neumann problem" of Spencer. They can be specified within the cohomology spaces of the complex (1.1).

Lemma 5.2. For every $i$, the natural mapping $\iota: \mathcal{H}_{N}^{i}(\mathcal{X}) \rightarrow H^{i}\left(C^{\infty}\left(\mathcal{X}, F^{\cdot}\right)\right)$ is injective.

Proof. Let $f \in \mathcal{H}_{N}^{i}(\mathcal{X})$ and $f=A y$ for some section $y \in C^{\infty}\left(\mathcal{X}, F^{i-1}\right)$. Then we get $(f, f)=\left(y, A^{*} f\right)=0$, i.e., $f=0$.

In 1963, Spencer suggested an approach that in certain cases allowed one to prove the surjectivity of the mapping $\mathcal{H}_{N}^{i}(\mathcal{X}) \rightarrow H^{i}\left(C^{\infty}\left(\mathcal{X}, F^{\cdot}\right)\right)$. It consists in establishing the normal solvability of the boundary value problem for solutions of $\Delta u=f$ in $\mathcal{X}$ subject to the boundary conditions $n(u)=0$ and $n(A u)=0$. We now turn to this approach.

Lemma 5.3. As defined above, $\mathcal{H}_{N}^{i}(\mathcal{X})$ coincides with the space of all sections $u \in C^{\infty}\left(\mathcal{X}, F^{i}\right)$ satisfying $\Delta u=0$ in $\mathcal{X}$ and $n(u)=0$ and $n(A u)=0$ on $\partial \mathcal{X}$.

Proof. If $u \in \mathcal{H}_{N}^{i}(\mathcal{X})$, then clearly $\Delta u=0$ in $\mathcal{X}$ and $n(u)=0$ and $n(A u)=0$ on $\partial \mathcal{X}$.

On the other hand, let a section $u \in C^{\infty}\left(\mathcal{X}, F^{i}\right)$ satisfy $\Delta u=0$ in $\mathcal{X}$ and $n(u)=0$ and $n(A u)=0$ on $\partial \mathcal{X}$. By Stokes' formula, we get $(\Delta u, u)=(A u, A u)+\left(A^{*} u, A^{*} u\right)$. Hence it follows that $A u=0$ and $A^{*} u=0$ in $\mathcal{X}$, as desired.

Given a smooth section $f$ of the bundle $F^{i}$, we consider the boundary value problem

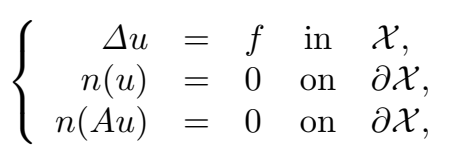

which is an obvious generalisation of the classical Neumann problem. This latter corresponds to the de Rham complex and $i=0$. It is worth pointing out that (5.1) fails to be an elliptic boundary problem in the case of general complexes (1.1), for the Lopatinskii condition is violated. In this section we discuss (5.1) via the $L^{2}$-approach.

It is easy to verify that (5.1) just amounts to the Euler equations for the functional

$$
u \mapsto \frac{1}{2}(A u, A u)+\frac{1}{2}\left(A^{*} u, A^{*} u\right)-(f, u)
$$

whose domain is the subspace of $C^{\infty}\left(\mathcal{X}, F^{i}\right)$ consisting of those $u$ which satisfy $n(u)=0$ on $\partial \mathcal{X}$. Motivated by this observation, we consider the so-called Dirichlet 
norm $D(u)=\left(\|A u\|^{2}+\left\|A^{*} u\right\|^{2}+\|u\|^{2}\right)^{1 / 2}$ on $C^{\infty}\left(\mathcal{X}, F^{i}\right)$ and denote by $\mathcal{D}_{L^{i}, N}$ the completion of the space of all $u \in C^{\infty}\left(\mathcal{X}, F^{i}\right)$ with $n(u)=0$ on $\partial \mathcal{X}$ under the norm $D(u)$. Since $D(u) \geq\|u\|$, we can identify $\mathcal{D}_{L^{i}, N}$ with a subspace of $L^{2}\left(\mathcal{X}, F^{i}\right)$. For $u \in \mathcal{D}_{L^{i}, N}$, the values $A u$ and $A^{*} u$ are well defined as elements of $L^{2}\left(\mathcal{X}, F^{i+1}\right)$ and $L^{2}\left(\mathcal{X}, F^{i-1}\right)$, respectively. Clearly, $A u$ and $A^{*} u$ are the differentials of $u$ in the weak sense in the interior of $\mathcal{X}$. Smooth elements $u$ of $\mathcal{D}_{L^{i}, N}$ inherit the property $n(u)=0$ on $\partial \mathcal{X}$.

Given $f \in L^{2}\left(\mathcal{X}, F^{i}\right)$, by a weak solution of (5.1) is meant any section $u \in \mathcal{D}_{L^{i}, N}$ satisfying

$$
(A u, A v)+\left(A^{*} u, A^{*} v\right)=(f, v)
$$

for all $v \in C^{\infty}\left(\mathcal{X}, F^{i}\right)$, such that $n(v)=0$ on $\partial \mathcal{X}$.

If $u \in \mathcal{D}_{L^{i}, N}$ fulfills (5.2), then $\Delta u=f$ weakly in the interior of $\mathcal{X}$, as is easy to see. Since $\Delta^{i}$ is elliptic, it follows that the "smoothness" of $u$ in the interior of $\mathcal{X}$ is two greater than that of $f$. In particular, $u$ is infinitely differentiable in the interior of $\mathcal{X}$ if so is $f$.

Lemma 5.4. If $u \in C^{\infty}\left(\mathcal{X}, F^{i}\right)$ is a weak solution of (5.1), then $u$ also satisfies (5.1) pointwise on $\mathcal{X}$.

Proof. See Lemma 4.1.16 of [Tar95].

Thus, if $f \in C^{\infty}\left(\mathcal{X}, F^{i}\right)$ and for a solution $u \in \mathcal{D}_{L^{i}, N}$ of (5.2) we could prove the regularity up to the boundary, then $u$ would be a classical solution of (5.1). Problem (5.1) is said to be (globally) hypoelliptic if, for every section $f \in C^{\infty}\left(\mathcal{X}, F^{i}\right)$, all the weak solutions $u \in \mathcal{D}_{L^{i}, N}$ of (5.1) are infinitely differentiable up to the boundary in $\mathcal{X}$.

Lemma 5.5. If (5.1) is hypoelliptic, then the space $\mathcal{H}_{N}^{i}(\mathcal{X})$ is of finite dimension.

Proof. As (5.1) is hypoelliptic, $\mathcal{H}_{N}^{i}(\mathcal{X})$ consists of all sections $u \in \mathcal{D}_{L^{i}, N}$ satisfying (5.2) with $f=0$. Consider two topologies $\tau_{1}$ and $\tau_{2}$ on $\mathcal{H}_{N}^{i}(\mathcal{X})$. The first of the two is induced from the $C^{\infty}\left(\mathcal{X}, F^{i}\right)$ topology. Endowed with this topology, $\mathcal{H}_{N}^{i}(\mathcal{X})$ is a Fréchet-Schwartz space. The topology $\tau_{2}$ is the topology on $\mathcal{H}_{N}^{i}(\mathcal{X})$ given by means of the Dirichlet norm $D(u)$. Under $\tau_{2}$, the space $\mathcal{H}_{N}^{i}(\mathcal{X})$ is easily seen to be Banach because $\left|(A u, A v)+\left(A^{*} u, A^{*} v\right)\right| \leq D(u) D(v)$ for all $u \in \mathcal{D}_{L^{i}, N}$ and $v \in C^{\infty}\left(\mathcal{X}, F^{i}\right)$. Since the topology $\tau_{1}$ is obviously stronger than the topology $\tau_{2}$, it follows from the open mapping theorem that these topologies on $\mathcal{H}_{N}^{i}(\mathcal{X})$ coincide. Therefore, as each normed Fréchet-Schwartz space is finite dimensional, the space $\mathcal{H}_{N}^{i}(\mathcal{X})$ is, too.

It is a general observation that, for the problem (5.1) to be solvable, it is necessary that $f$ should be orthogonal to the space of solutions of the homogeneous boundary value problem on $\mathcal{X}$ that is formally adjoint to the Neumann problem with respect to the Green formula for the Laplacian. However, Lemma 2.1 implies that the "Neumann problem" of Spencer for the complex (1.1) is actually formally selfadjoint relative to the Green formula. It follows that, for (5.1) to be solvable, it is necessary that

$$
f \perp \mathcal{H}_{N}^{i}(\mathcal{X})
$$


Corollary 5.6. If problem (5.1) is solvable under condition (5.3), then a necessary and sufficient condition for solvability of the inhomogeneous equation $A y=f$ is $A f=0$ and $f \perp \mathcal{H}_{N}^{i}(\mathcal{X})$.

The crucial result on the solvability of (5.1) is due to Kohn and Nirenberg, see [KN65. They proved that if the inclusion $\mathcal{D}_{L^{i}, N} \hookrightarrow L^{2}\left(\mathcal{X}, F^{i}\right)$ is compact, then the space $\mathcal{H}_{N}^{i}(\mathcal{X})$ is finite dimensional, (5.2) is solvable under condition (5.3), and (5.2) is hypoelliptic.

Here we restrict our discussion to solvability of (5.1) in infinitely differentiable sections of $F^{i}$. To this end, for any $i$ we introduce the so-called Neumann space $\mathcal{N}^{i}(\mathcal{X})$ consisting of all $u \in C^{\infty}\left(\mathcal{X}, F^{i}\right)$ which satisfy $n(u)=0$ and $n(A u)=0$ on $\partial \mathcal{X}$.

Theorem 5.7. Suppose that the inclusion $\mathcal{D}_{L^{i}, N} \hookrightarrow L^{2}\left(\mathcal{X}, F^{i}\right)$ is compact. Then there exist bounded operators $H_{N}^{i}$ and $N^{i}$ in $L^{2}\left(\mathcal{X}, F^{i}\right)$ such that

1) $H_{N}^{i}$ is the $L^{2}$-orthogonal projection onto the subspace of harmonic sections $\mathcal{H}_{N}^{i}(\mathcal{X})$;

2) $N^{i}$ is a bounded operator from $L^{2}\left(\mathcal{X}, F^{i}\right)$ to $\mathcal{D}_{N}^{i}$ mapping $C^{\infty}\left(\mathcal{X}, F^{i}\right)$ to $\mathcal{N}^{i}(\mathcal{X})$ and satisfying $H_{N}^{i} N^{i}=N^{i} H_{N}^{i}=0$;

3) $f=H_{N} f+A^{*} A N f+A A^{*} N f$ fulfills weakly in the interior of $\mathcal{X}$ for all $f \in L^{2}\left(\mathcal{X}, F^{i}\right)$.

Proof. See for instance Proposition 4.1.26 of [Tar95].

If $A f=0$, then $A N f=0$. If moreover both $N^{i}$ and $N^{i+1}$ are defined, one easily verifies that actually $A^{i} N^{i}=N^{i+1} A^{i}$ holds. It follows that, under the hypothesis of Theorem 5.7 the natural mapping $\mathcal{H}_{N}^{i}(\mathcal{X}) \rightarrow H^{i}\left(C^{\infty}\left(\mathcal{X}, F^{\cdot}\right)\right)$ is a topological isomorphism.

Since $A^{N-1}$ has surjective symbol, the inclusion $\mathcal{D}_{L^{N}, N} \hookrightarrow L^{2}\left(\mathcal{X}, F^{N}\right)$ is compact. Thus, the hypothesis of Theorem 5.7 is always satisfied in the extreme case $i=N$. On the contrary, the inclusion $\mathcal{D}_{L^{0}, N} \hookrightarrow L^{2}\left(\mathcal{X}, F^{0}\right)$ may not be compact, for the space $\mathcal{H}_{N}^{0}(\mathcal{X})$ is usually infinite dimensional for compact manifolds with boundary. One invokes the operator $N^{1}$ to study the "Neumann problem" of Spencer at step 0, see [Tar95, 4.2.8].

For a thorough treatment of the operator $N$, called the Neumann operator, we refer the reader to $\S 4.2$ of [Tar95].

\section{Dirichlet to Neumann operator}

For any $0 \leq i \leq N-1$, the Dirichlet to Neumann operator $\Psi$ on $C^{\infty}\left(\partial \mathcal{X}, F_{t}^{i}\right)$ is defined as follows. lem

Given any $u_{0} \in C^{\infty}\left(\partial \mathcal{X}, F_{t}^{i}\right)$, Theorem 4.2 shows that the boundary value prob-

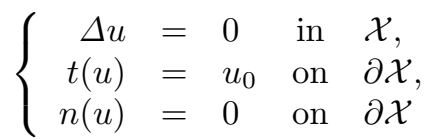

has at least one solution $u \in C^{\infty}\left(\mathcal{X}, F^{i}\right)$. The solution of (6.1) is unique up to a harmonic section of $F^{i}$ of the Hodge theory developed in $\$ 3$ i.e., up to an element of $\mathcal{H}^{i}(\mathcal{X})$. Set $\Psi u_{0}=\imath \ell n(A u)$ on $\partial \mathcal{X}$. By the above, $\Psi u_{0} \in C^{\infty}\left(\mathcal{X}, F_{t}^{i}\right)$ is independent of the choice of the solution $u$, and so $\Psi$ is a well-defined operator on $C^{\infty}\left(\partial \mathcal{X}, F_{t}^{i}\right)$. 
In the case $i=0$, our definition just amounts to the classical one, for the second boundary condition in (6.1) is automatically fulfilled. We also set $\Psi^{N}=0$, for the bundle $F_{t}^{N}$ is trivial.

From what has been said at the end of $\$ 4$ it follows that $\Psi u_{0}=\imath \ell n\left(A P\left(u_{0}, 0\right)\right)$, where $P$ is the Poisson operator of Corollary 4.3. This formula readily highlights the pseudodifferential nature of the Dirichlet to Neumann operator. More precisely, we get $\Psi^{i} \in \Psi^{1}\left(F_{t}^{i}\right)$ and the principal symbol of $\Psi^{i}$ may be evaluated in the usual manner.

Lemma 6.1. As defined above, the Dirichlet to Neumann operator $\Psi$ is nonnegative in the sense that

$$
\int_{\partial \mathcal{X}}\left(\Psi u_{0}, u_{0}\right)_{x} d s \geq 0
$$

for all $u_{0} \in C^{\infty}\left(\partial \mathcal{X}, F_{t}^{i}\right)$.

Proof. Let $u_{0}, v_{0} \in C^{\infty}\left(\partial \mathcal{X}, F_{t}^{i}\right)$. Choose solutions $u, v \in C^{\infty}\left(\mathcal{X}, F^{i}\right)$ to (6.1) with data $u_{0}$ and $v_{0}$, respectively. By the Green formula used in the proof of Lemma 5.1, we get

$$
\begin{aligned}
(A u, A v) & =\left(u, A^{*} A v\right)+\int_{\partial \mathcal{X}}(t(u), \imath \ell n(A v))_{x} d s \\
\left(A^{*} u, A^{*} v\right) & =\left(u, A A^{*} v\right)-\int_{\partial \mathcal{X}}\left(\iota \ell n(u), t\left(A^{*} v\right)\right)_{x} d s
\end{aligned}
$$

whence

$$
\int_{\partial \mathcal{X}}\left(u_{0}, \Psi v_{0}\right)_{x} d s=(A u, A v)+\left(A^{*} u, A^{*} v\right) .
$$

Setting $v_{0}=u_{0}$ yields the lemma.

Since each nonnegative closed operator with dense domain in a complex Hilbert space is selfadjoint, cf. [Sch01, 12.11], Lemma 6.1] gives rise to a selfadjoint extension of $\Psi$ in $L^{2}\left(\partial \mathcal{X}, F_{t}^{i}\right)$. We only state the formal selfadjointness of $\Psi$, which can be seen immediately.

Corollary 6.2. For each $i$, the Dirichlet to Neumann operator $\Psi$ is formally selfadjoint, i.e.,

$$
\int_{\partial \mathcal{X}}\left(u_{0}, \Psi v_{0}\right)_{x} d s=\int_{\partial \mathcal{X}}\left(\Psi u_{0}, v_{0}\right)_{x} d s
$$

for all $u_{0}, v_{0} \in C^{\infty}\left(\partial \mathcal{X}, F_{t}^{i}\right)$.

Proof. Given $u_{0}, v_{0} \in C^{\infty}\left(\partial \mathcal{X}, F_{t}^{i}\right)$, let $u, v \in C^{\infty}\left(\mathcal{X}, F^{i}\right)$ be solutions to the boundary value problem (6.1) with data $u_{0}$ and $v_{0}$, respectively. The Green formula of Lemma 2.1 yields

$$
\int_{\partial \mathcal{X}}\left(\left(u_{0}, \Psi v_{0}\right)_{x}-\left(\Psi u_{0}, v_{0}\right)_{x}\right) d s=0,
$$

which establishes the formula.

We are now able to characterise the null space of the Dirichlet to Neumann operator on $C^{\infty}\left(\partial \mathcal{X}, F_{t}^{i}\right)$.

Lemma 6.3. For $u_{0} \in C^{\infty}\left(\partial \mathcal{X}, F_{t}^{i}\right)$ to satisfy $\Psi u_{0}=0$, it is necessary and sufficient that there be a $u \in \mathcal{H}_{N}^{i}(\mathcal{X})$, such that $t(u)=u_{0}$ on $\partial \mathcal{X}$. 
Proof. Write Ker $\Psi^{i}$ for the null space of $\Psi$ acting in $C^{\infty}\left(\partial \mathcal{X}, F_{t}^{i}\right)$. We claim that $\operatorname{Ker} \Psi^{i}=t\left(\mathcal{H}_{N}^{i}(\mathcal{X})\right)$, the space of the tangential parts on $\partial \mathcal{X}$ of all harmonic sections in $\mathcal{H}_{N}^{i}(\mathcal{X})$. Indeed, if $u_{0} \in \operatorname{Ker} \Psi^{i}$ and $u$ is a solution to (6.1), then $t(u)=u_{0}, n(u)=0$ and

$$
\begin{aligned}
(A u, A u)+\left(A^{*} u, A^{*} u\right) & =\int_{\partial \mathcal{X}}\left(u_{0}, \Psi u_{0}\right)_{x} d s \\
& =0
\end{aligned}
$$

by (6.2). This means that $u \in \mathcal{H}_{N}^{i}(\mathcal{X})$. Conversely, if $u_{0}=t(u)$ for $u \in \mathcal{H}_{N}^{i}(\mathcal{X})$, then $u$ is a solution to the boundary value problem (6.1), and so $\Psi u_{0}=\imath \ell n(A u)$ vanishes, as desired.

The range Ran $\Psi^{i}$ of the Dirichlet to Neumann operator acting in $C^{\infty}\left(\partial \mathcal{X}, F_{t}^{i}\right)$ fails to be closed in the general case. As a counterexample, we show the Dirichlet to Neumann operator at step 0 for the Dolbeault complex on the closure of a bounded domain in $\mathbb{C}^{n}$.

\section{Betti nUmbers}

If we know the null space of $\Psi^{i}$, we can derive some low bounds for the dimension of the cohomology of (1.1) at step $i$. We call this latter the $i$-th Betti number of the complex (1.1).

Theorem 7.1. As defined in 96 , the Dirichlet to Neumann operator possesses the property that

$$
\operatorname{Ker} \Psi^{i} \stackrel{\text { top. }}{\cong} \frac{\mathcal{H}_{N}^{i}(\mathcal{X})}{\mathcal{H}^{i}(\mathcal{X})} \text {. }
$$

Note that $\mathcal{H}^{i}(\mathcal{X})$ is a closed subspace of $\mathcal{H}_{N}^{i}(\mathcal{X})$, and so their quotient space is separated.

Proof. To shorten notation, denote by $Q$ the quotient space on the right-hand side of (7.1). Consider the mapping $\iota: Q \rightarrow \operatorname{Ker} \Psi^{i}$ given by $\iota[u]:=t(u)$ for $u \in \mathcal{H}_{N}^{i}(\mathcal{X})$, where $[u]$ stands for the equivalence class of $u$ in $Q$. This definition is correct, for $t(u) \in \operatorname{Ker} \Psi^{i}$ fulfills by Lemma 6.3, and if $u_{1}, u_{2} \in \mathcal{H}_{N}^{i}(\mathcal{X})$ belong to the same class in $Q$, then their difference $u_{1}-u_{2}$ lies in $\mathcal{H}^{i}(\mathcal{X})$, whence $t\left(u_{1}-u_{2}\right)=0$. If $\iota[u]=0$ for some $u \in \mathcal{H}_{N}^{i}(\mathcal{X})$, then $t(u)=0$ on $\partial \mathcal{X}$ and so $u$ actually belongs to $\mathcal{H}^{i}(\mathcal{X})$. Hence $[u]=0$, showing that the mapping $\iota$ is injective. On the other hand, Lemma 6.3 implies the surjectivity of $\iota$. Since the continuity of $\iota$ is obvious, the theorem follows.

As is mentioned in 93 , the space $\mathcal{H}^{i}(\mathcal{X})$ is of finite dimension. On the other hand, the natural mapping $\mathcal{H}_{N}^{i}(\mathcal{X}) \rightarrow H^{i}\left(C^{\infty}\left(\mathcal{X}, F^{\cdot}\right)\right)$ is injective, which is due to Lemma 5.2. We thus get

$$
\begin{aligned}
\operatorname{dim} \operatorname{Ker} \Psi^{i} & =\operatorname{dim} \mathcal{H}_{N}^{i}(\mathcal{X})-\operatorname{dim} \mathcal{H}^{i}(\mathcal{X}) \\
& \leq \operatorname{dim} H^{i}\left(C^{\infty}\left(\mathcal{X}, F^{\cdot}\right)\right)-\operatorname{dim} \mathcal{H}^{i}(\mathcal{X}),
\end{aligned}
$$

showing that the $i$-th Betti number of (1.1) exceeds $\operatorname{dim} \operatorname{Ker} \Psi^{i}+\operatorname{dim} \mathcal{H}^{i}(\mathcal{X})$ for all $i=0,1, \ldots, N$.

We now turn to the theory of Spencer developed in $\$ 5$ to see that in most reasonable situations the $i$-th Betti number of (1.1) just amounts to the dimension 
of $\operatorname{Ker} \Psi^{i}$. By "most reasonable" are actually meant the cases where the cohomology of (1.1) is proved to be finite dimensional at step $i$. This requires certain convexity assumptions on $\mathcal{X}$.

Corollary 7.2. Suppose that the inclusion $\mathcal{D}_{L^{i}, N} \hookrightarrow L^{2}\left(\mathcal{X}, F^{i}\right)$ is compact. Then it follows that

$$
\operatorname{dim} H^{i}\left(C^{\infty}\left(\mathcal{X}, F^{\cdot}\right)\right)=\operatorname{dim} \operatorname{Ker} \Psi^{i}+\operatorname{dim} \mathcal{H}^{i}(\mathcal{X}) .
$$

Proof. If the inclusion $\mathcal{D}_{L^{i}, N} \hookrightarrow L^{2}\left(\mathcal{X}, F^{i}\right)$ is compact, then, by Theorem 5.7, the natural mapping $\mathcal{H}_{N}^{i}(\mathcal{X}) \rightarrow H^{i}\left(C^{\infty}\left(\mathcal{X}, F^{\cdot}\right)\right)$ is an isomorphism. It remains to use Theorem 7.1 .

For $i=0$ the corollary is still true if "the inclusion $\mathcal{D}_{L^{i}, N} \hookrightarrow L^{2}\left(\mathcal{X}, F^{i}\right)$ is compact" is deleted from the hypotheses. Indeed, the cohomology $H^{0}\left(C^{\infty}\left(\mathcal{X}, F^{*}\right)\right)$ reduces obviously to $\mathcal{H}_{N}^{0}(\mathcal{X})$. In the other extreme case $i=N$ this hypothesis can also be removed, for $\mathcal{D}_{L^{N}, N}=\mathcal{D}_{L^{N}}$ and the inclusion $\mathcal{D}_{L^{N}} \hookrightarrow L^{2}\left(\mathcal{X}, F^{N}\right)$ is always compact.

Example 7.1. Consider the Dolbeault complex with coefficients in a holomorphic vector bundle $F$ over a compact strictly pseudoconvex manifold $\mathcal{X}$ of dimension $n$. In this case we use the designation $\Omega \cdot(\mathcal{X}, F)$ for the complex (1.1). Corollary 7.2 states that $\operatorname{dim} H^{i}(\Omega(\mathcal{X}, F))=\operatorname{dim} \operatorname{Ker} \Psi^{i}$ is valid for all $i=0,1, \ldots, n$, because $\mathcal{H}^{i}(\mathcal{X})$ is zero by the uniqueness theorem for analytic functions. Thus, the Hodge numbers of the manifold $\mathcal{X}$ manifest themselves through the Dirichlet to Neumann operator introduced above.

Whether $\mathcal{H}^{i}(\mathcal{X})$ is trivial or not depends on the uniqueness property of the local Cauchy problem for $\Delta^{i}$. In particular, $\mathcal{H}^{i}(\mathcal{X})$ is zero in case all the objects are real analytic.

\section{ACKNOWLEDGEMENTS}

The author acknowledges financial support of the DFG (German Research Society), grant TA 289/4-1.

\section{REFERENCES}

[Bel03] Belishev, M. I., The Calderon problem for two-dimensional manifolds by the BC-method, SIAM J. Math. Anal. 35 (2003), no. 1, 172-182. MR2001471 (2004f:58029)

[Bel05] Belishev, M. I., Some remarks on the impedance tomography problem for $3 D$-manifolds, CUBO A Math. J. 7 (2005), no. 1, 42-55. MR2140807 (2006j:58035)

[BSh08] Belishev, M., and Sharafutdinov, V., Dirichlet to Neumann operator on differential forms, Bull. des Sci. Math. 132 (2008), 128-145. MR2387822 (2008m:58045)

[BC10] Bellassoued, M., and Choulli, M., Stability estimate for an inverse problem for the magnetic Schrödinger equation from the Dirichlet-to-Neumann map, J. Funct. Anal. 258 (2010), no. 1, 161-195. MR 2557958

[Hyv09] Hyvönen, N., Comparison of idealized and electrode Dirichlet-to-Neumann maps in electric impedance tomography with an application to boundary determination of conductivity, Inverse Problems 25 (2009), no. 8, 085008, 18 pp. MR2529198

[KSU07] Kenig, C. E., Sjöstrand, J., and Uhlmann, G., The Calderón problem with partial data, Ann. of Math. (2) 165 (2007), no. 2, 567-591. MR2299741 (2008k:35498)

[KN65] Kohn, J. J., and Nirenberg, L., Non-coercive boundary value problems, Comm. Pure Appl. Math. 18 (1965), 443-492. MR0181815 (31:6041)

[LU01] Lassas, M., and Uhlmann, G., On determining the Riemannian manifold from the Dirichlet-to-Neumann map, Ann. Sci. Ecole Norm. Super. 34 (2001), no. 4, 771-787. MR 1862026 (2003e:58037) 
[LTU03] Lassas, M., Taylor, M., and Uhlmann, G., The Dirichlet to Neumann map for complete Riemannian manifolds with boundary, Comm. Anal. Geom. 11 (2003), no. 2, 207-221. MR2014876 (2004h:58033)

[LU89] Lee, J., and Uhlmann, G., On determining anisotropic real analytic conductivities by boundary measurements, Comm. Pure Appl. Math. 42 (1989), 1097-1112. MR.1029119 (91a:35166)

[Mor56] Morrey, C. B., A variational method in the theory of harmonic integrals. II, Amer. J. Math. 78 (1956), no. 1, 137-170. MR0087765 (19:408a)

[NPT07] Nachman, A. I., Päivärinta, L., and Teirilä, A., On imaging obstacles inside inhomogeneous media, J. Funct. Anal. 252 (2007), no. 2, 490-516. MR2360925 (2008k:78024)

[NTT09] Nachman, A. I., Tamasan, A., and Timonov, A., Recovering the conductivity from a single measurement of interior data, Inverse Problems 25 (2009), no. 3, 035014, 16 pp. MR 2480184 (2010g:35340)

[Roi96] Roitberg, Ya. A., Elliptic Boundary Value Problems in Generalized Functions, Kluwer Academic Publishers, Dordrecht NL, 1996. MR.1423135 (97m:35055)

[Sch60] Schechter, M., Negative norms and boundary problems, Ann. of Math. (2) 72 (1960), no. 3, 581-593. MR0125333 (23:A2636)

[Sch01] Schechter, M., Principles of Functional Analysis, 2nd ed., American Mathematical Society, Providence, RI, 2001. MR.1861991 (2002j:46001)

[SST03] Schulze, B.-W., Shlapunov, A., and Tarkhanov, N., Green Integrals on Manifolds with Cracks, Annals of Global Analysis and Geometry 24 (2003), no. 2, 131-160. MR.1990112 (2004i:58041)

[Tar95] Tarkhanov, N., Complexes of Differential Operators, Kluwer Academic Publishers, Dordrecht, NL, 1995. MR1368856 (96k:58205)

[Tay96] Taylor, M., Partial Differential Operators II. Qualitative Studies of Linear Equations, Springer-Verlag, New York, Inc., 1996. MR.1395149 (98b:35003)

Institut für Mathematik, Universität Potsdam, Am Neuen Palais 10, 14469 Potsdam, Germany

E-mail address: tarkhanov@math.uni-potsdam.de 